\title{
FINDING FREQUENT SUBPATHS IN A GRAPH
}

\author{
Sumanta Guha \\ Computer Science \& Information Management Program \\ Asian Institute of Technology \\ P.O. Box 4, Klong Luang \\ Pathumthani 12120 \\ Thailand
}

\begin{abstract}
The problem considered is that of finding frequent subpaths of a database of paths in a fixed undirected graph. This problem arises in applications such as predicting congestion in network and vehicular traffic. An algorithm, called AFS, based on the classic frequent itemset mining algorithm Apriori is developed, but with significantly improved efficiency over Apriori from exponential in transaction size to quadratic through exploiting the underlying graph structure. This efficiency makes AFS feasible for practical input path sizes. It is also proved that a natural generalization of the frequent subpaths problem is not amenable to any solution quicker than Apriori.
\end{abstract}

\section{KEYWORDS}

AFS, Apriori, data mining, frequent subpath, frequent sub-structure, graph mining

\section{INTRODUCTION}

Within the general problem of mining frequent patterns from a database of transactions, an area of some recent interest is where the transactions occur in a structured or semi-structured set. The structure considered often is that of a graph because objects of interest in various applications can, in fact, be modeled as graphs, e.g., chemical compounds, web links, virtual communities, XML specifications and networks of different kinds.

Finding frequent subgraphs of a database of graph transactions has been of particular interest. Algorithms for this problem based on Apriori (the classic frequent itemset mining algorithm due to Agrawal \& Srikant [1]) have been given, amongst others, by Kuramochi \& Karypis [13], Inokuchi et al. [10] and Vanetik et al. [14], while Yan \& Han [15] give an algorithm which uses a novel encoding scheme for graphs. More recently, big graph - a graph so large it cannot fit in the memory (primary or secondary) of one machine - mining is emerging as an area of particular importance because of its application in understanding social networks. The algorithm of Jha et al. [11] to count triangles in a big graph whose data is streaming in on-line is typical of big graph mining. Kang \& Faloutsos [12] describe software for big graph mining. See Cook \& Holder [5] and Han \& Kamber [9] for a survey of general graph mining techniques. 
The problem which we consider is a particular case of the problem of finding frequent subgraphs. In particular, in our case all transactions are paths in a fixed undirected graph, and we are interested in determining those paths in that graph which occur frequently as subpaths of the transaction paths. This is a natural problem to consider. For example, if each path in the database represents the route taken by an object such as a message or vehicle, then the frequent subpaths represent congested sections, or hot spots. Related work includes Chen et al. [3] and Gudes \& Pertsev [7], which both compute the paths themselves that are frequently traversed, but are based on hashing and pruning techniques for which the authors only present empirical evidence of efficiency, while our approach, based on Apriori, is entirely different and provably efficient.

A simple-minded application of Apriori to finding frequent subpaths - say, by treating paths as itemsets - falls short because the feasibility of Apriori depends on transactions being of small size. However, paths in graphs arising from practical applications are not necessarily short (e.g, consider vehicular traffic in a city) and a straight Apriori-type solution runs into exponential complexity. Instead, we exploit the graph structure for a significant gain in efficiency which leads to a generally applicable solution, which we call AFS (Apriori for Frequent Subpaths).

In Section 2 we specify both the problem and its solution by the AFS algorithm. In Section 3 we analyze and compare the complexities of Apriori and AFS in finding frequent subpaths to prove a theoretical gain in efficiency from exponential in input size of the former to low polynomial of the latter. Section 4 discusses experimental verification of our theoretical claims, as well as an application of AFS to text mining.

In Section 5 we show that, interestingly, there is no possibility of similarly leveraging the graph structure to improve Apriori for a solution to a natural generalization of the frequent subpaths problem - that of finding so-called frequent strings of subpaths - because the general problem is equivalent in complexity to that of finding frequent itemsets. We conclude in Section 6.

\section{Problem AND ALgorithm}

\subsection{Problem Statement}

Let $\mathrm{G}=(\mathrm{V}, \mathrm{E})$ be an undirected graph with vertex set $\mathrm{V}$ and edge set $\mathrm{E}$.

Here are some definitions related to paths in graphs that we'll use. A path $\mathrm{P}$ in $\mathrm{G}$ of length $k$ from a vertex $u$ to $u^{\prime}$ is a sequence $\left(v_{0}, v_{1}, \ldots, v_{k}\right)$ of vertices such that $v_{0}=u$ and $v_{k}=u^{\prime}$ and $\left(v_{i-1}, v_{i}\right) \in$ E for $i=1,2, \ldots, k$. (We'll also allow the empty sequence () to denote the empty path of undefined length.) A path $Q$ in $G$ is said to be a subpath of $P$, denoted $Q \triangleright P$, if $Q=\left(w_{0}, w_{1}, \ldots\right.$ ,$\left.w_{k^{\prime}}\right)$, where $\left(w_{0}, w_{1}, \ldots, w_{k^{\prime}}\right)$ is a contiguous subsequence of $\left(v_{0}, v_{l}, \ldots, v_{k}\right)$, i.e., if, for some $i$ such that $0 \leq i \leq i+k^{\prime} \leq k$, we have $w_{0}=v_{i}, w_{l}=v_{i+1}, \ldots, w_{k^{\prime}}=v_{i+k^{\prime}}$. In this case, if $i=0$ then $\mathrm{Q}$ is called a prefix subpath of $\mathrm{P}$; if $i+k^{\prime}=k$ then $Q$ is called a suffix subpath of $\mathrm{P}$. For a non-empty path $\mathrm{P}=(v 0, v 1, \ldots, v k)$, front $(P)$ denotes the first vertex $v_{0}$ and $\operatorname{tail}(P)$ denotes the suffix subpath $\left(v_{l}, \ldots, v_{k}\right)$. A path (or, subpath) of length $k$ will often be called a $k$-path (or, $k$-subpath).

Following are a couple of more database-related definitions to do with paths. Let $P$ be a given set of paths in $\mathrm{G}$. A path $\mathrm{Q}$ in $\mathrm{G}$ is said to have support support $(\mathrm{Q})=|\{\mathrm{P} \in P: \mathrm{Q} \triangleright \mathrm{P}\}|$, i.e., the 
International Journal of Data Mining \& Knowledge Management Process (IJDKP) Vol.4, No.5, September 2014 number of paths in $P$ of which $\mathrm{Q}$ is a subpath. Moreover, suppose a minimum support value min_sup is specified. If support $(\mathrm{Q}) \geq \min \_$sup, then $Q$ is said to be a frequent subpath.

The statement of the problem is now straightforward: Given a set $P$ of paths in an undirected graph $\mathrm{G}$, determine all frequent subpaths.

See Figure 1 for an example of three paths in a grid graph.

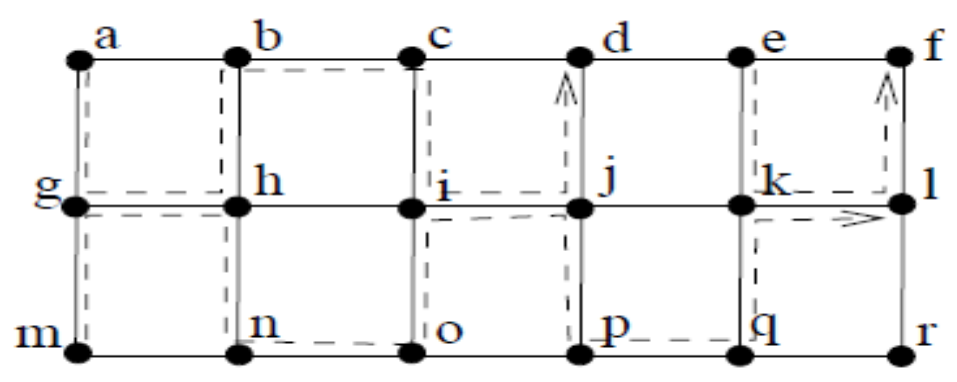

Fig. 1. A grid graph with three paths indicated by directed broken lines. If $\min \_s u p=2$ then the frequent subpaths are $(g),(h),(i),(j),(k),(l),(g, h),(i, j)$ and $(k, l)$.

Remark: In database terminology, $P$ is a database of transactions, where each transaction $\mathrm{P}$ is a path in a fixed graph $\mathrm{G}$.

\subsection{Apriori Algorithm}

As our algorithm to find frequent subpaths is derived from Apriori, the classic frequent itemset mining algorithm due to Agrawal \& Srikant [1], and as we'll be comparing the complexities of the two, we'll first specify Apriori somewhat exactly.

Let $\mathcal{D}$ be a database of transactions, where each transaction $\mathrm{T} \in D$ is a subset of a set of all items $\mathcal{J}$. The support of an itemset $\mathrm{I} \subset \mathcal{J}$. is support $(\mathrm{I})=|\{\mathrm{T} \in \mathrm{D}: \mathrm{I} \subset \mathrm{T}\}|$. If support (I) $\geq$ min_sup, then $I$ is frequent. Following is pseudo-code for the Apriori algorithm to determine all frequent itemsets (adapted from Agrawal \& Srikant [1]).

\section{$\underline{\text { Apriori }}$}

$L_{1}=\{$ frequent 1-itemsets $\}$;

for $\left(k=2 ; L_{k-1} \neq \emptyset ; k++\right)$

\{

$C_{k}=$ join $\left(L_{k-1}, L_{k-1}\right) ; / /$ Generate candidates.

$C_{k}=\operatorname{prune}\left(C_{k}\right)$; // Prune candidates

$L_{k}=\operatorname{checkSupport}\left(C_{k}\right)$; // Eliminate candidate

// if support too low.

\}

return $U_{k} L_{k}$;// Returns all frequents itemsets. 
We discuss next the routines in the Apriori for loop and how all three are implemented using a function subset $(X, T)$ - where $\mathrm{X}$ is a set of itemsets and $\mathrm{T}$ is an itemset - which returns the subset $\mathrm{Y}$ of $\mathrm{X}$ consisting of those itemsets which are contained in $\mathrm{T}$. We'll discuss implementing $\operatorname{subset}(X, T)$ itself later.

Firstly, join $\left(L_{k-1}, L_{k-1}\right)$ generates all $k$-itemsets of the form $\left\{i_{1}, i_{2}, \ldots, i_{k}\right\}$, where both $\left\{i_{1}, i_{2}, \ldots\right.$, $\left.i_{k-1}\right\}$ and $\left\{i_{1}, i_{2}, \ldots, i_{k-2}, i_{k}\right\}$ belong to $L_{k-1}$ (note that itemsets are always assumed listed in lexicographic order), i.e., unions of pairs of itemsets in $L_{k-1}$ both of whose members share the same first $k-2$ items. Secondly, prune $\left(C_{k}\right)$ deletes all $I \in C_{k}$ such that some $(k-1)$-subset of $I$ does not belong to $L_{k-1}$. It may be checked that both join $\left(L_{k-1}, L_{k-1}\right)$ and prune $\left(C_{k}\right)$ are implemented by the following routine which uses subset $\left(L_{k-1}, *\right)$ :

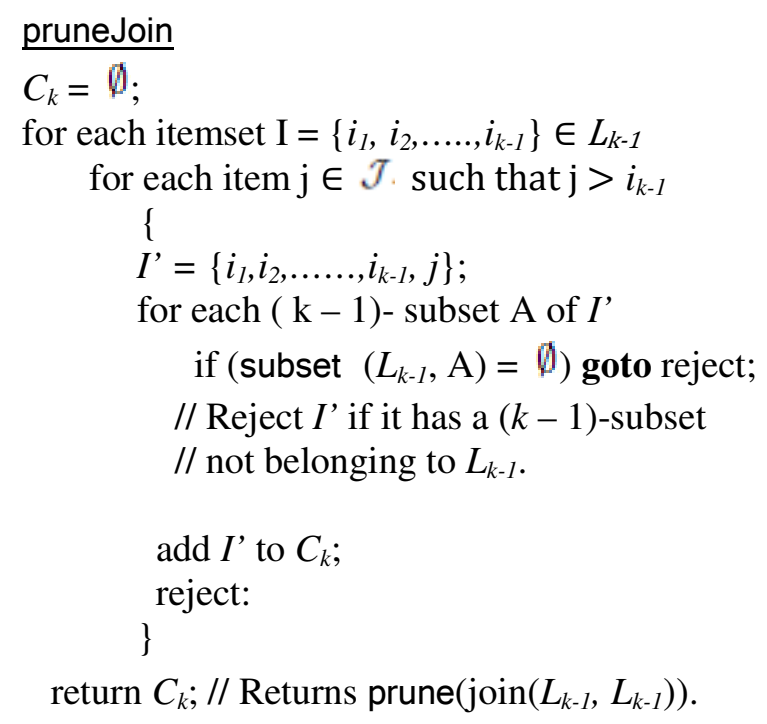

Finally, checkSupport $\left(C_{k}\right)$ counts the support of each itemset currently in $C_{k}$ to eliminate those that are not frequent. It is straightforwardly implemented with the help of subset $\left(\mathrm{C}_{\mathrm{k}},{ }^{*}\right)$ :

checkSupport

$L_{k}=\emptyset$;

for each $\mathrm{I} \in C_{k}$

I. count $=0$;

for each transaction $T \in D$

\{

$C_{T}=\operatorname{subset}\left(C_{k}, \mathrm{~T}\right)$;

for each $I \in C_{T}$

I.count++;

\}

for each $\mathrm{I} \in C_{k}$

if (I.count $\geq$ min_sup) add $I$ to $L_{k}$;

return $L_{k}$;// Returns members of $C_{k}$ with support

// at least min_sup. 
Therefore, when implementing Apriori the calls to join and prune in the for loop are replaced by a single call to pruneJoin, while checkSupport is implemented as above.

The function subset $(\mathrm{X}, \mathrm{T})$ is implemented by first storing the itemsets of $\mathrm{X}$ in a trie (prefix tree as in Fredkin [6]) $\mathcal{T}$ on the "alphabet" $\mathcal{J}$ of items ordered lexicographically, each itemset treated as an ordered string. Agrawal \& Srikant[1] actually use a particular implementation called a hash tree as developed by Coffman Jr. \& Eve [4], where pointers to children are stored in a hash table keyed on items at each internal node (the use of a hash tree in this case instead of a simple trie is justified by the typically large size of $\mathcal{J}$ ). See Figure 2 for an example.

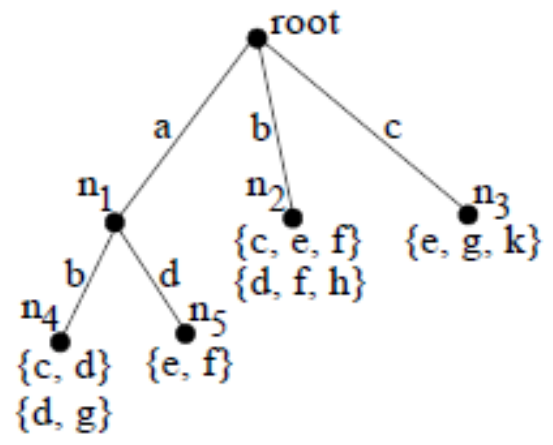

Fig.2. A hash tree $\mathcal{T}$ storing a set of six 4-itemsets $X=\{\{a, b, c, d\},\{a, b, d, g\},\{a, d, e, f\},\{b, c, e, f\},\{b, d, f, h\}$, $\{\mathrm{c}, \mathrm{e}, \mathrm{g}, \mathrm{k}\}\}$, where each leaf can store at most two itemsets (only the suffix of an itemset following the prefix defined by the path to the leaf is stored).

The function subset $(\mathrm{X}, \mathrm{T})$ is then executed by calling $\operatorname{doSubset}(\operatorname{root}(\mathcal{T}), \mathrm{T})$ using the recursive routine below:

doSubset(node, I)

\{

$\mathrm{Y}=\emptyset$;

if (node is leaf) add checkltemsets (node, I) to Y ;

// Function checkltemsets(node, I) returns those

$/ /$ itemsets stored at node that are contained in I.

else if $(I=\emptyset)$; // Nothing is added to $Y$.

else for each $(i \in \mathrm{I})$

if (node.ch( $i$ ) exists)

add $i * \operatorname{doSubset}($ node.ch( $i),\{j \in \mathrm{I}: j>i\})$ to $\mathrm{Y}$;

// For each item $i \in$ I recurse on the corresponding

$/ /$ child of node. We denote by $i * \mathrm{Z}$ the union of $i$

// with each itemset in $\mathrm{Z}$.

\section{return $\mathrm{Y}$;}

\} 
For example, in Figure 2, $\operatorname{doSubset}($ root, $\{a, b, c, d, e, f\})$ makes three recursive calls to doSubset with parameters $\left(n_{1},\{b, c, d, e, f\}\right),\left(n_{2},\{c, d, e, f\}\right)$ and $(\mathrm{n} 3,\{\mathrm{~d}, \mathrm{e}, \mathrm{f}\})$, respectively. The first of these in turn calls doSubset with parameters $(n 4,\{c, d, e, f\})$ and (n5, $\{e, f\})$, while the second and third add $\{b, c, e, f\}$ and nothing, respectively, to the answer $\mathrm{Y}$, etc.

Though various technical improvements in implementing Apriori have been suggested - see Han \& Kamber [9] for a discussion - we'll not consider them here, but use as our reference the basic implementation described above.

\subsection{AFS: Apriori for Frequent Subpaths}

We present our algorithm AFS (Apriori for Frequent Subpaths) in as similar a manner as possible to that for Apriori in the previous section so that it's easy to see exactly how the added structure in the setting of AFS helps make it more efficient.

$\underline{\mathrm{AFS}}$

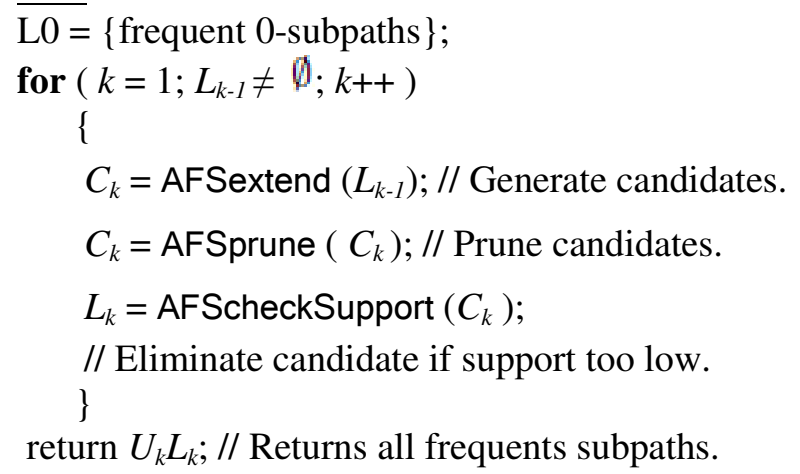

The gain from the graph structure is first seen in generating candidates: we obtain $C_{k}$ by simply extending each path in $\left.L_{k-1}\right)$. This is justified as it may be seen that the set of k-paths obtained by so extending paths in $L_{k-l}$ indeed contains $L_{k}$. Pruning is simpler as well because, after extending a path $\mathrm{P}$ in $L_{k-1}$ to a $k$-path P', the only ( $\mathrm{k}-1$ )-subpath of P' whose membership in $L_{k-1}$ need be checked is its suffix $\mathrm{k}-1$-subpath. The reason is that $\mathrm{P}$ ' has only two $\mathrm{k}-1$-subpaths: one prefix ( $P$ itself ) and the other suffix.

E.g., in Figure 1, $(g, h) \in L_{1}$ would generate four extensions for inclusion in $C_{2}:(g, h, i),(g, h, b)$, $(g, h, g)$ and $(g, h, n)$. Moreover, in the prune step, e.g., for $(g, h, i)$, only $(h, i)$ has to be checked if it belongs to $L_{l}$.

Both AFSextend $\left(L_{k-1}\right)$ and $\operatorname{AFSprune}\left(C_{k}\right)$ are implemented by the routine AFSpruneExtend below, which should be compared with the earlier pruneJoin routine for Apriori. AFSpruneExtend uses the function subpaths $(\mathrm{X}, \mathrm{P})$, where $\mathrm{X}$ is a set of paths and $\mathrm{T}$ is a path, which returns the subset $\mathrm{Y}$ of $\mathrm{X}$ consisting of those paths which are subpaths of $\mathrm{T}$ (function subpaths $(X, P)$, whose implementation we'll detail momentarily, is, of course, the counterpart of the earlier subset $(\mathrm{X}, \mathrm{T})$ ). 


\section{AFSpruneExtend}

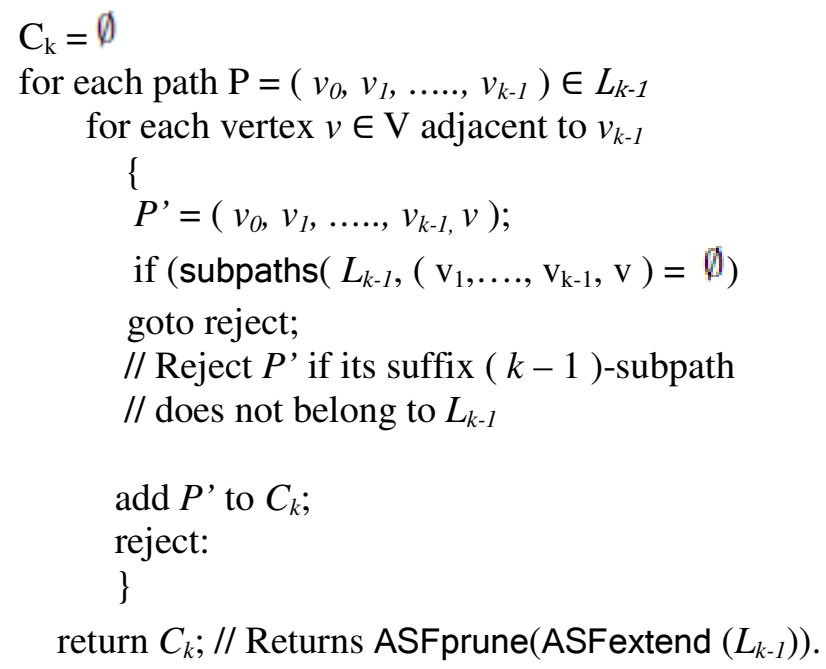

The routine AFScheckSupport is a near copy of its Apriori counterpart checkSupport.

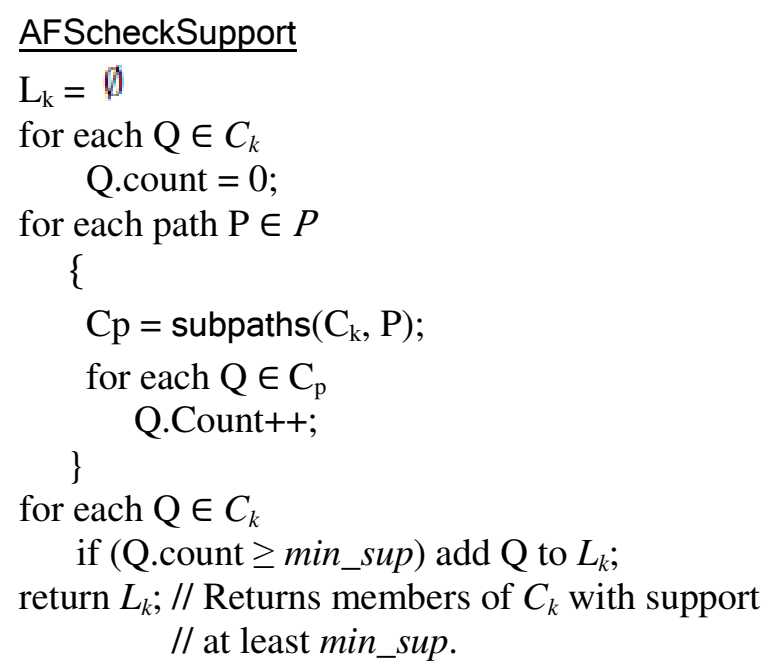

Therefore, when implementing AFS the calls to AFSextend and AFSprune in the for loop are replaced by a single call to AFSpruneExtend, while AFScheckSupport is implemented as above.

It's in implementing subpaths $(\mathrm{X}, \mathrm{P})$ that we leverage the graph setting of AFS to huge gain over subset(X, T ) (we'll see the actual calculations in the next section). Paths in $\mathrm{X}$ are stored in a hash tree $\mathcal{T}$ as well, exactly as for subset(X, T ). It's straightforward to use this tree of paths to determine which are prefix subpaths of $\mathrm{P}$. Therefore, noting that a path in $\mathrm{X}$ is a subpath of $\mathrm{P}$ if an only if it is a prefix subpath of some suffix subpath of $\mathrm{P}$, subpaths $(\mathrm{X}, \mathrm{P})$ is implemented by calling doSubpaths $\left(\operatorname{root}(\mathcal{T}),\left(w_{o}, w_{l}, \ldots . w_{k}\right)\right)$, where $P=\left(w_{o}, w_{l}, \ldots, w_{k}\right)$. 
doSubpaths $\left(\right.$ node,$\left.\left\{w_{0}, w_{1}, \ldots . w_{k}\right\}\right)$

\{

$\mathrm{Y}=\emptyset$;

for $(\mathrm{i}=0 ; \mathrm{i} \leq \mathrm{k} ; \mathrm{i}++)$

add doPrefixSubpaths $\left(\operatorname{node},\left(w_{i}, w_{i+1}, \ldots \ldots, w_{k}\right)\right)$

to $\mathrm{Y}$;

// Iteratively calls doPrefixSubpaths $($ node, $\mathrm{Q}) / /$ on each suffix of $\mathrm{Q}$ of $\mathrm{P}=\left(w_{0}, w_{1}, \ldots, w_{k}\right)$.

return $\mathrm{Y}$

\}

Compare the following with doSubset.

doPrefixSubpaths (node, $Q$ )

\{

$\mathrm{Y}=\emptyset$;

if (node is leaf) add checkPrefixPaths (node, $\mathrm{Q}$ ) to $\mathrm{Y}$;

// Function checkPrefixPaths (node, Q) returns those

$/ /$ paths stored at node that are prefix subpaths of $\mathrm{P}$.

else if ( $\mathrm{Q}=())$; // Nothing is added to $\mathrm{Y}$.

else

if (node.ch(first $(\mathrm{Q}))$ exists)

add $\operatorname{first}(\mathrm{Q})$ *

doPrefixSubpaths $($ node.ch $(\operatorname{first}(\mathrm{Q}))$, $\operatorname{tail}(\mathrm{Q}))$ to $\mathrm{Y}$;

// Descend from node along the path labeled by

$/ /$ successive vertices of $\mathrm{Q}$. We denote by $v * \mathrm{Z}$ the

$/ /$ concatenation of $v$ with each path in $\mathrm{Z}$.

return Y;

\}

For example, suppose the hash tree in Figure 2 represents a set of paths instead of itemsets. Then, the call doSubpaths (node, $(a, b, c, d, e, f)$ ) spawns six iterations of the call doPrefixSubpaths with parameters (node, $(a, b, c, d, e, f)),($ node,$(b, c, d, e, f)), \ldots,($ node, $(f))$, respectively. Each of the doPrefixSubpaths calls descends recursively from the root down a single path of $\mathcal{T}$ E.g., the one with parameters (node, $(a, b, c, d, e, f))$ descends to $\mathrm{n} 4$ to finally call doPrefixSubpaths $(\mathrm{n} 4,(c, d$, $e, f)$ ), which adds $(a, b, c, d)$ to the answer $\mathrm{Y}$. 


\section{ANALYSIS : AFS VS. APRIORI}

Consider Apriori first. The recursion in $\operatorname{doSubset}($ node, $I)$ yields a Fibonacci-type recurrence in running time of $t(k)=t(k-1)+t(k-2)+\ldots t(1)$, if $\mathrm{I}=\left\{i_{1}, i_{2}, \ldots, i_{k}\right\}$, implying a time bound function of order exponential in the size of $\mathrm{I}$, which we indicate by $\mathrm{O}(\exp (\mathrm{III}))$ (We ignore the cost of calls to checkltemsets $($ node,$I)$.) The size of the hash tree rooted at node is an obvious upper time bound as well on doSubset(node, I).

Therefore, similar bounds apply to $\operatorname{subset}(\mathrm{X}, \mathrm{T})$ as well. In particular, $\operatorname{subset}\left(C_{k}, \mathrm{~T}\right)$ and subset $\left(L_{k}, \mathrm{~T}\right)$, used to implement Apriori, are bounded in running time by $\mathrm{O}(\min (\exp (\mathrm{IT} \mathrm{I})$, size $\left.\left.\mathrm{ht}\left(C_{k}\right)\right)\right)$ and $\mathrm{O}\left(\min \left(\exp (\mathrm{IT} \mathrm{I})\right.\right.$, size_ht $\left.\left.\left(L_{k}\right)\right)\right)$, respectively, where size $\mathrm{ht}(\mathrm{X})$ denotes the size of the hash tree storing $X$.

It follows that the total time cost incurred by calls to pruneJoin from Apriori is

$$
O\left(|\mathcal{J}| \sum_{k} k\left|L_{k}\right| \min \left(\exp (k), \operatorname{size} \_h t\left(L_{k}\right)\right)\right)
$$

(the expectation that on the average there will be $O(|\mathcal{J}|)$ items greater than the last one in an itemset justifies the $|\mathcal{J}|$ factor) and by those to checkSupport is

$$
O\left(\sum_{k}\left(\left|C_{k}\right|+\sum_{T \in \mathcal{D}} \min \left(\exp (|T|), \operatorname{size} \_h t\left(C_{k}\right)\right)\right)\right.
$$

Next, consider AFS. The routine doPrefixSubpaths (node, $Q$ ) is bounded by time linear in $|Q|$ as the recursion descends from node along a path labeled by successive vertices of $Q$. The height height of the hash tree rooted at node is a bound as well. Consequently, doSubpaths (node, $P$ ) takes time $\mathrm{O}(\min (\mid \mathrm{PI}$, height $)+\min (|\mathrm{P}|-1$, height $)+\ldots+\min (1$, height $))=\mathrm{O}\left(\min \left(|\mathrm{P}|^{2},|\mathrm{P}|\right.\right.$ height $\left.)\right)$.

Therefore, subpaths $\left(C_{k}, P\right)$ runs in time bounded by $\mathrm{O}\left(\min \left(|\mathrm{P}|^{2}\right.\right.$, $|\mathrm{P}|$ height_ht $\left.\left(C_{k}\right)\right)$ ), and subpaths $\left(L_{k}, P\right)$ in time bounded by $\mathrm{O}\left(\min \left(|\mathrm{P}|^{2}\right.\right.$, $|\mathrm{P}|$ height_ht $\left.\left.\left(L_{k}\right)\right)\right)$, where height_ht $(\mathrm{X})$ denotes the height of the hash tree storing $X$, which represents a gain in efficiency over the corresponding Apriori routine subset(X,T) from exponential to quadratic.

We have, therefore, that the total time cost incurred by calls to AFSextendJoin from AFS is

$$
O\left(\sum_{k}\left|L_{k}\right| \min \left(k^{2} \text {, height_ht }\left(L_{k}\right)\right)\right)
$$

(We assume that on the average each vertex has $\mathrm{O}(1)$ neighbors) and those to AFScheckSupport is

$$
O\left(\sum_{k}\left(\left|C_{k}\right|+\sum_{P \in \mathcal{P}} \min \left(|P|^{2}, \text { height_ht }\left(C_{k}\right)\right)\right)\right.
$$


Clearly, Apriori is vulnerable to exponential time worst-case behavior. In fact, it's evident from the complexity expressions for pruneJoin and checkSupport that the feasibility of applying Apriori lies in assuming that (a) the size of individual transactions in the database is $\mathrm{O}(1)$, and (b) the size of $\mathrm{C}_{\mathrm{k}}$ decreases rapidly with $\mathrm{k}$. Fortunately, both assumptions are justified in various practical scenarios, e.g., market basket analysis.

In case of AFS though (a) is not a reasonable assumption: transactions in the database, i.e., paths in a graph, may not be short, or $\mathrm{O}(1)$, in length. In practical applications, e.g., vehicles traveling in a network of roads, paths taken may even be of size comparable to that of the graph itself. However, we see from the last two expressions above that, even then, AFS has a worst-case behavior quadratic in the total length of the input paths, making it practically applicable.

\section{EXPERIMENTAL VERIFICATION AND AN APPLICATION}

The theoretical advantage of AFS can be tested practically by generating random sets of paths in large graphs, and then finding frequent subpaths using both Apriori (ignoring the graph structure and treating paths as itemsets of vertices) and AFS.

Ali [2], in fact, does exactly this following a preliminary description of AFS in an earlier conference paper (Guha [8]). He generates random sets of paths in grid graphs of maximum dimension $5 \times 20$ and complete graphs with a maximum of 25 nodes, applying both Apriori and AFS to find frequent subpaths. His results demonstrate clearly the nearly combinatorial explosion in running time of the former algorithm versus the far slower growth rate of the latter.

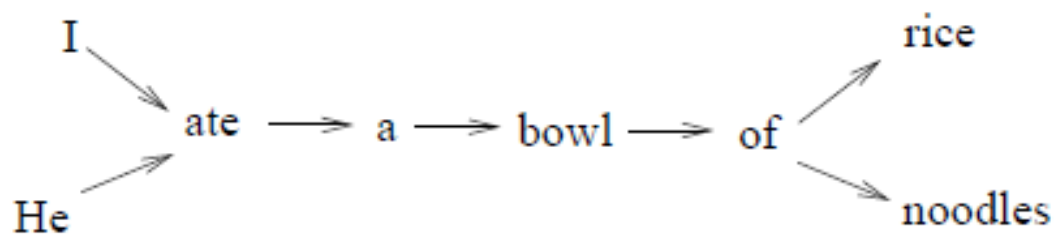

Fig. 3. Paths in a graph of words.

Ali also shows a nice application of AFS to text mining, in particular, mining frequent phrase patterns in the Arabic Quranic text corpus. The idea is explained in Figure 3: the common phrase ate a bowl of of the two word paths I ate a bowl of rice and He ate a bowl of noodles is found as a subpath of both. Ali then uses these frequent phrases to index and cluster Quranic verses.

\section{A Generalization AND its HaRdNESS}

The intersection of a set of paths in an undirected graph $\mathrm{G}$ is not necessarily a path, but a union of paths. We'll call such an intersection a string of subpaths, or, simply, string. Therefore, a natural generalization of the frequent subpaths problem considered in the previous section is as follows: Given a set $P$ of paths in an undirected graph $G$, determine all frequent strings of subpaths.

For example, in Figure 1, $(\mathrm{g}, \mathrm{h}) \cup(\mathrm{i}, \mathrm{j})$ and $(\mathrm{k}, \mathrm{l})$ are the two maximal frequent strings. Observe that knowing all frequent strings evidently implies knowing all frequent subpaths. However, the converse is not true -e.g., it's not possible to deduce from the fact that $(\mathrm{g}, \mathrm{h}) \cup(\mathrm{i}, \mathrm{j})$ is a frequent 
string. Therefore, the problem of finding frequent strings is at least as hard as that of finding frequent subpaths.

Surely, an Apriori-type algorithm may be implemented to find all frequent strings, but, interestingly, no improvement in efficiency over Apriori (as in AFS) can be expected because, as we'll see momentarily, the problem of finding frequent itemsets is equivalent to that of finding frequent strings. Firstly, we'll reduce the first problem to the second in time linear in the size of the input.

Let $\mathrm{D}$ be a database of transactions, each transaction $\mathrm{T}$ being a subset of the set of all items $\mathcal{J}$. Let $\mathrm{G}$ be the complete graph on the set of vertices $\mathrm{V}=\mathcal{J}$. Represent each transaction $\mathrm{T} \in \mathrm{D}$. where $\mathrm{T}=\left\{i_{1}, i_{2}, \ldots ., i_{k}\right\}$, by the path $\mathrm{P}_{\mathrm{T}}=\left(i_{1}, i_{2}, \ldots \ldots, i_{k}\right)$, the items in $\mathrm{T}$ being in lexicographic order. It may be seen that, given the set of paths $P=\left\{P_{T}: T \in D\right\}$, the set of frequent strings corresponds exactly to the set of frequent itemsets for the database $\mathrm{D}$, which completes the reduction claimed and proves that finding frequent strings is atleast as hard as finding frequent itemsets.

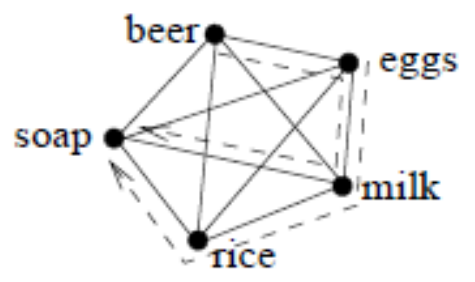

Fig. 4. The database of two transactions \{beer, eggs, milk, soap\} and \{eggs, milk, rice, soap\} over the set of items $\mathcal{J}=$ \{beer, eggs, milk, rice, soap $\}$ is represented by two corresponding paths in the complete graph on $\mathcal{J}$.

E.g., for the database of Figure 4, if $\min \sup =2$, then the one maximal frequent itemset is \{eggs, milk, soap $\}$ and the corresponding one maximal frequent string is (eggs, milk) $\cup$ (soap).

We omit details of the fairly straightforward reduction in the opposite direction. The equivalence of the two problems means that there is no hope of leveraging the graph structure to find a more efficient variation of Apriori to determine frequent strings. However, this should not be an issue in practical applications where it is enough to simply identify the congested subpaths.

\section{CONCLUSION}

We have developed the AFS algorithm to find frequent subpaths which, though derived from Apriori, exploits the underlying graph structure for a gain in efficiency that makes it applicable to practical input sizes for this particular problem. We believe that similar improvements may be found for related problems, e.g., finding frequent subtrees of a collection of trees. The development of a general framework in which to place the problem of finding frequent substructures of a collection of structures belonging to a family with certain given inheritance properties (e.g., a subgraph of a path is a union of paths) would be significant as well.

Given the emergence of big graph mining, it would be useful to be able to find frequent subpaths from streaming input data a la Jha et al. [11] who count triangles of an input graph. 
International Journal of Data Mining \& Knowledge Management Process (IJDKP) Vol.4, No.5, September 2014

\section{REFERENCES}

[1] R. Agrawal, R. Srikant, Fast algorithms for mining association rules, VLDB'94, Proceedings of 20th International Conference on Very Large Data Bases (1994), 487-499.

[2] I. Ali, Application of a Mining Algorithm to Finding Frequent Patterns in a Text Corpus: A Case Study of the Arabic, International Journal of Software Engineering and Its Applications 6 (2012), 127-134.

[3] M. S. Chen, J. S. Park, P. S. Yu, Efficient Data Mining for Path Traversal Patterns, IEEE Transactions on Knowledge and Data Engineering 10 (1998), 209-221.

[4] E. G. Coffman Jr., J. Eve, File structures using hashing functions, Communications of the ACM 13 (1970), 427-432.

[5] D. J. Cook, L. B. Holder, Mining Graph Data, Wiley Interscience, 2006.

[6] E. Fredkin, Trie memory, Communications of the ACM 3 (1960), 490-499.

[7] E. Gudes, A. Pertsev, Mining module for adaptive XML path indexing, Proceedings of the 16th International Workshop on Database and Expert Systems Applications (2005), 1015-1019.

[8] S. Guha, Efficiently Mining Frequent Subpaths, Proceedings of the Eighth Australasian Data Mining Conference (AusDM 2009) (2009), 11-15.

[9] J. Han,M. Kamber, Data Mining Concepts and Techniques, 2nd Edition, Morgan Kaufmann, 2005.

[10] A. Inokuchi, T. Washio, H. Motoda, An Apriori-based Algorithm for Mining Frequent Substructures from Graph Data, Proceedings of the 4th European Conference on Principles of Data Mining and Knowledge Discovery (Lecture Notes In Computer Science 1910 (2000), 12-23

[11] M. Jha, C. Seshadri, A. Pinar, A Space Efficient Streaming Algorithm for Triangle Counting using the Birthday Paradox, 19th ACM SIGKDD International Conference on Knowledge Discovery and Data Mining (KDD’13) (2013), 589-597.

[12] U. Kang, C. Faloutsos, Big Graph Mining: Algorithms and Discoveries, ACM SIGKDD Explorations 14 (2012), 29-36.

[13] M. Kuramochi, G. Karypis, Frequent Subgraph Discovery, Proceedings of the 2001 IEEE International Conference on Data Mining (2001), 313-320.

[14] N. Vanetik, E. Gudes, S. E. Shimony, Computing Frequent Graph Patterns from Semistructured Data, Proceedings of the 2002 IEEE International Conference on Data Mining (2002), 458-465.

[15] X. Yan, J. Han, gSpan: Graph-Based Substructure Pattern Mining, Proceedings of the 2001 IEEE International Conference on Data Mining (2002), 721-724.

\section{AUTHOR}

Sumanta Guha obtained his Ph.D. in Computer Science from the University of Michigan, Ann Arbor, in 1991. From 1991 to 2002 he taught at the University of Wisonsin-Milwaukee as a member of the Electrical Engineering \& Computer Science faculty. Since 2002 he has been with the Computer Science \& Information Management Program at the Asian Institute of Technology, Thailand, where he is a professor.

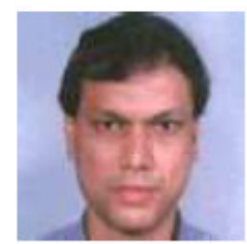

